\title{
ARTICLE \\ Glutamatergic and gabaergic ventral BNST neurons differ in their physiological properties and responsiveness to noradrenaline
}

\author{
Nur Zeynep Gungor ${ }^{1}$, Ryo Yamamoto ${ }^{1}$ and Denis Pare ${ }^{1}$
}

\begin{abstract}
The bed nucleus of the stria terminalis (BNST) regulates defensive responses to threats and its anteroventral portion (BNST-AV) is involved. BNST-AV contains a minority of glutamatergic neurons scattered among a dominant population of GABAergic cells. There is evidence that these two cell types might exert opposite influences, the former promoting and the latter reducing anxiety. Although GABAergic cells greatly outnumber glutamatergic neurons in BNST-AV, in some circumstances the influence of glutamatergic cells appears to predominate. Related to this, BNST-AV receives a very strong noradrenaline (NA) input and negative emotional states are associated with a marked rise of NA concentration in BNST-AV. However, it is currently unclear whether NA differentially alters the excitability of glutamatergic and GABAergic BNST-AV neurons. Thus, to shed light on how BNST-AV regulates negative emotional states, the present study compared the physiological properties and NA responsiveness of glutamatergic and GABAergic BNST-AV neurons using whole-cell recordings in transgenic mice that express a fluorescent reporter in either cell group. We found that glutamatergic cells had a slightly more complex morphology than the GABAergic cells, a higher intrinsic excitability, and a different responsiveness to NA. Indeed, while NA inhibited EPSPs in both cell types through a1 and a2 adrenoreceptors, the EPSP reduction seen in glutamatergic cells had a lower amplitude and a shorter duration than in GABAergic cells. These differences were due to the presence of a $\beta$-receptor-mediated EPSP enhancement in the glutamatergic cells. Together, our results suggest that multiple properties contribute to the disproportionate influence of glutamatergic BNST-AV neurons.
\end{abstract}

Neuropsychopharmacology (2018) 43:2126-2133; https://doi.org/10.1038/s41386-018-0070-4

\section{INTRODUCTION}

The bed nucleus of the stria terminalis (BNST) is not only involved in anxiety-like responses to unpredictable threats [1] but also regulates fear responses to discrete threatening cues [2]. This regulation depends on complex interactions between several cell groups in BNST. Among them are CRF-expressing neurons found in the anterolateral region of BNST, particularly its oval nucleus, which are thought to mediate the anxiogenic influence of BNST [3]. Although CRF-expressing BNST neurons have received much attention, there is evidence that glutamatergic cells in anteroventral portion of BNST (BNST-AV) also promote negative emotional states. In BNST-AV, a small group of glutamatergic neurons, estimated to account for $1-3 \%$ of the cells, is scattered among a dominant population of GABAergic cells ([4, 5]; but see ref. [6] for higher estimates), and it is proposed that the two cell types exert opposite influences on negative emotional states. Indeed, while glutamatergic BNST-AV neurons increase their firing rates during aversive stimuli, GABAergic BNST-AV cells show inhibitory responses to the same stimuli [7]. Consistent with these results, optogenetically activating glutamatergic BNST-AV cells and adjacent regions causes place aversion and anxiogenic effects, whereas activation of GABAergic cells produces place preference and decreased anxiety [7].

Paralleling the above, there is also evidence that these two cell groups also regulate the HPA-axis in opposite ways. For instance, selective immunotoxic lesions of GABAergic cells increase adrenocorticotropic hormone and corticosterone levels after restraint stress [8], indicating that GABAergic cells tonically inhibit the HPA axis. Supporting this interpretation, lesions of structures that provide excitatory inputs to BNST-AV, like the medial prefrontal cortex [8] and hippocampus [9], decrease the number of fospositive GABAergic cells in BNST-AV, while increasing fos expression in the paraventricular hypothalamic nucleus (PVN). Yet, nonselective lesions that affect both cell types reduce stress-induced fos expression in the PVN ([10-12]; however see [13]). Despite the limitations of lesion studies, these observations together raise the possibility that the impact of glutamatergic cells outweighs that of the more numerous GABAergic cells, such that BNST-AV exerts an overall excitatory influence on the HPA axis.

Importantly, BNST-AV's influence over negative emotional states is regulated by a massive noradrenaline (NA) input, one of the densest in the brain $[14,15]$. Aversive events like immobilization stress [16, 17], predator odors [18], formalin-induced pain [19] and bitter solutions [20] increase NA levels in BNST-AV. While NA was reported to strongly inhibit BNST-AV neurons in vivo [21] and in vitro [22], glutamatergic and GABAergic neurons could not be distinguished in these earlier studies.

Determining whether NA differentially regulates these two cell types through different receptor sub-types might allow us to reconcile discrepant findings in the literature. Specifically, while

${ }^{1}$ Center for Molecular and Behavioral Neuroscience, Rutgers University, Newark, NJ 07102, USA

Correspondence: Nur Zeynep Gungor (ngungor@scarletmail.rutgers.edu) or Denis Pare (pare@andromeda.rutgers.edu)

Received: 4 December 2017 Revised: 5 April 2018 Accepted: 10 April 2018

Published online: 20 April 2018 
Vglut2-ires-Cre-Ai6 mouse
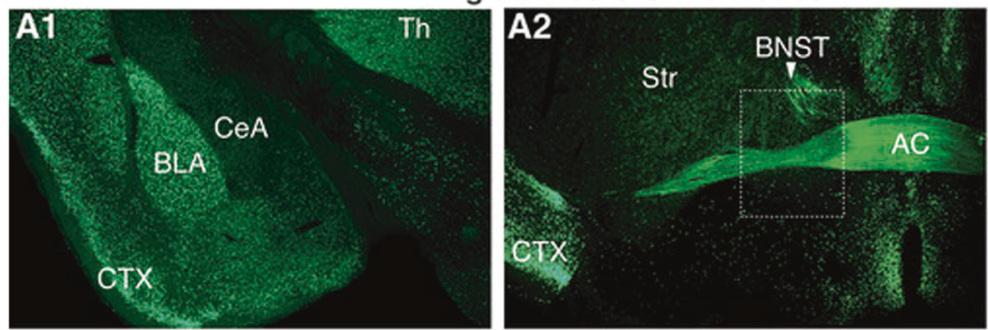

Vgat-ires-Cre-Ai6 mouse
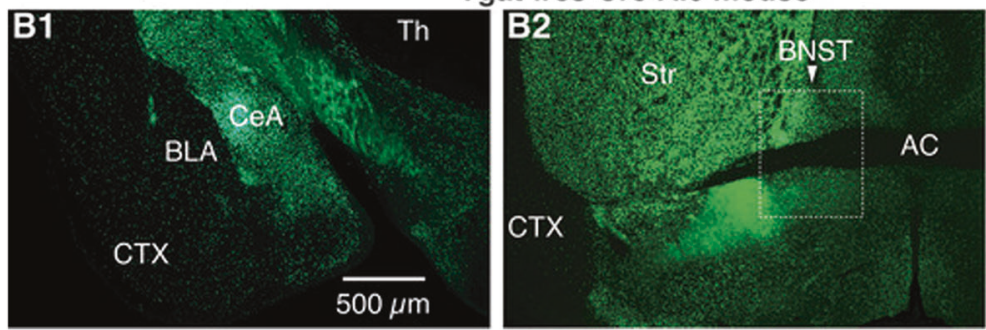

Fig. 1 Contrasting the distribution of Vglut2-reporter-positive (A) and Vgat-reporter-positive (B) neurons in the amygdala (A1,B1) and BNST (A2,B2). A Vglut2-Cre-IRES-knockin mice crossed with Ai6 reporter mice. B Vgat-Cre-IRES-knockin mice crossed with Ai6 reporter mice. A3,B3 BNST region at higher magnification. Ai6 mice have a targeted mutation of the Gt(ROSA)26Sor locus with a loxP-flanked STOP cassette preventing transcription of the enhanced green fluorescent protein ZsGreen1. Because ZsGreen1 is expressed following Cre-mediated recombination, we could separately visualize glutamatergic or GABAergic neurons in the two mice crosses. $\mathrm{AC}$ anterior commissure, $\mathrm{AL}$ anterolateral sector of BNST, AV anteroventral sector of BNST, BLA basolateral complex of the amygdala, CeA central nucleus of the amygdala, CTX cortex, Str striatum, Th thalamus

electrophysiological studies reported that $\beta A R$ agonists have no effect on BNST-AV neurons [22], beta-adrenergic receptor ( $\beta A R$ ) antagonist infusions in BNST-AV attenuates stress-induced reinstatement of cocaine-seeking [50], pain-induced place aversion [19] and anxiety-like behaviors [17]. Thus, it remains possible that glutamatergic cells mediate the behavioral influence of $\beta$ ARs and that these effects were undetected in physiological studies because they mostly sampled the prevalent population of GABAergic cells.

Thus, to shed light on how glutamatergic and GABAergic BNSTAV cells regulate negative emotional states, we compared their physiological properties and NA responsiveness using whole-cell recordings in transgenic mice that express a fluorescent reporter in either cell group.

\section{MATERIALS AND METHODS}

Procedures were approved by the Institutional Animal Care and Use Committee of Rutgers University, in compliance with the Guide for the Care and Use of Laboratory Animals (DHHS). One hundred and fifty nine mice of either sex (Jackson Laboratories, Farmington, CT) were used. To visualize glutamatergic or GABAergic BNST neurons, we crossed Vglut2-ires-Cre mice (Stock 016963) or Vgat-ires-Cre knock-in mice (Stock 016962) with Ai6 reporter mice (Stock 007906). Consistent with prior observations [23], sections from the Vglut2-ires-Cre-Ai6 and Vgat-ires-Cre-Ai6 mice looked like negative images of each other and the expression of the reporter fluorescent protein ZsGreen1 conformed to prior reports on the distribution of GABAergic and glutamatergic neurons in the brain ([5]; see Fig. 1 for amygdala and BNST). While we acknowledge that false positives and negatives in expression are unavoidable in transgenic lines, the large sample sizes used in this study should limit their influence.

Characterization of physiological properties

Methods for the preparation of coronal BNST slices, whole-cell recordings and data processing described previously [24] were adapted with minor modifications (Supplementary Methods). To assess spontaneous firing, cells were observed for $1 \mathrm{~min}$ at rest. To characterize their electroresponsive properties, we applied rectangular current pulses of gradually increasing amplitudes $( \pm 10 \mathrm{pA}$ increments; $500 \mathrm{~ms} ; 0.2 \mathrm{~Hz}$ ) from -55 to $-70 \mathrm{mV}$. Spike latency, firing threshold, and discharge rates were assessed at rheobase. Spike threshold was determined visually by examining the first and second derivatives of voltage traces.

\section{Histology}

To study the morphology of recorded cells, $0.5 \%$ biocytin was added to the pipette solution. The method used to reveal biocytin described in Rodriguez-Sierra et al. [25] was modified for thick tissue processing (Supplementary Methods). Labeled neurons were photographed and traced with a Nikon Eclipse E800 microscope using $\times 20, \times 40$, and $\times 100$ objectives and StereoInvestigator 8.0 (Microbrightfield Bioscience, Williston, VT). The distance from the soma periphery to tip of the longest process was measured to determine process extent. The total number of branches was defined as the sum of the secondary, tertiary, and quaternary branches.

\section{Stimulation and pharmacological analyses}

To activate synaptic inputs, a pair of tungsten stimulating electrodes (inter-tip spacing, $200 \mu \mathrm{m}$ ) was placed in the stria terminalis (ST) to deliver current pulses $(0.1 \mathrm{~ms} ; 0.05 \mathrm{~Hz})$ whose amplitude $(0.1-0.8$ $\mathrm{mA}$ ) was adjusted below orthodromic spiking threshold. During these tests, cells were kept at $-55 \mathrm{mV}$ and their input resistance (Rin) was monitored by applying $-10 \mathrm{pA}$ pulses $10 \mathrm{~s}$ before ST stimuli. A 10-min baseline was obtained before drug application. Antagonists were added to the perfusate $10 \mathrm{~min}$ before agonist application and were present throughout the recordings. Agonists were applied for $10 \mathrm{~min}$. In all experiments, picrotoxin $(100 \mu \mathrm{m})$ was added to the perfusate to suppress inhibitory transmission.

NA was aliquoted and stored at $-80^{\circ} \mathrm{C}$ with $100 \mu \mathrm{M}$ ascorbic acid to prevent oxidization. Other drugs were aliquoted and kept in -20 ${ }^{\circ} \mathrm{C}$. NA (N5785) was obtained from Sigma (St. Louis, MO). Isoproterenol (1747), Propranolol (0624), Phenylephrine (2838), and UK 14304 (2466), were obtained from R\&D Systems (Minneapolis, $\mathrm{MN})$. Drug concentrations were based on prior investigations of NA effects in BNST neurons and other brain regions. 

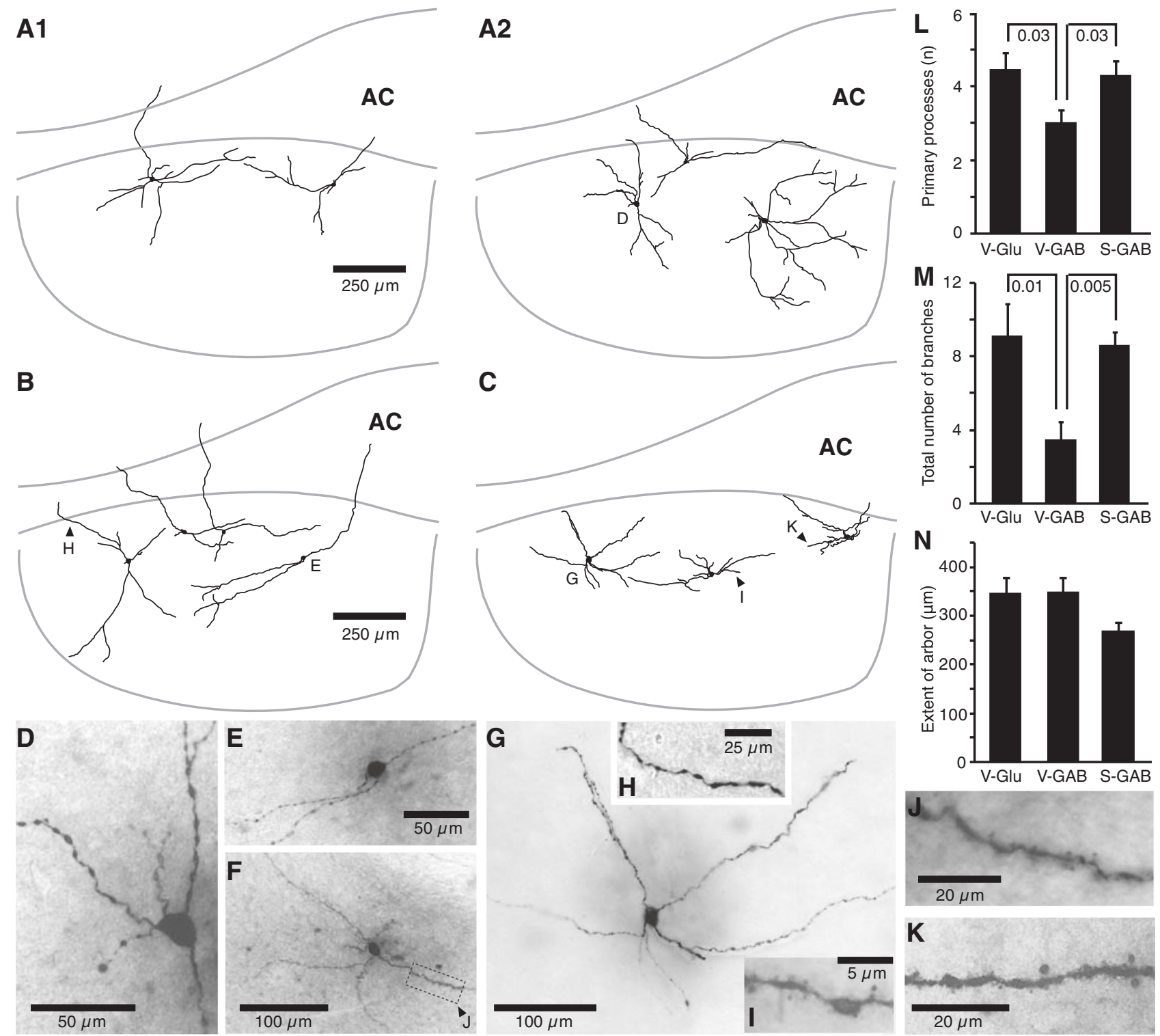

Fig. 2 Morphological properties of Vglut2-reporter-positive and Vgat-reporter-positive BNST-AV neurons. Camera lucida drawings of A Vglut2-reporter-positive or B Vgat-reporter-positive BNST-AV neurons bearing dendritic varicosities and C of Vgat-reporter-positive neurons with dendritic spines. Some of the cells depicted in A-C are labeled with letters. These letters refer to panels (shown below), which depict photomicrographs of the same neurons at a higher magnification. D, E Soma and proximal dendrites of varicose Vglut2-reporter-positive (D) and Vgat-reporter-positive (E) cells. F, G Low power view of spiny Vgat-reporter-positive cells. H-K Higher power depictions of $\mathbf{H}$ varicose dendrite, I spiny and varicose dendrite, and $\mathbf{K}$ spiny dendritic segment devoid of varicosities. L-N Bar graphs comparing the number of primary processes $F(2,28)=4.8, p=0.02$, one-way independent-samples ANOVA $(\mathbf{L})$, total number of branches $F(2,28)=6.8, p=0.004(\mathbf{M})$, and process extent $\mathrm{F}(2,28)=2.6, p=0.1(\mathrm{~N})$ in varicose Vglut2-reporter-positive (V-Glu), varicose Vgat-reporter-positive (V-GAB), and spiny Vgatreporter-positive cells. We report Bonferroni-Holm corrected $p$-values for post-hoc independent samples $t$-tests

Experimental design and statistical analyses

Data are reported as averages \pm SEM. Statistical tests are two-sided. After an initial screening for recording quality and stability done blind to data values, all cells were included in the statistical analyses. Approximately equal number of males and females were used. To check whether the distribution of data differed between sexes, the data was inspected statistically (using independent samples $t$-tests) or visually (when sample sizes were small). However, we observed that values overlapped extensively in all the experiments. Two brain sections containing BNST-AV were obtained from each animal. In each, we obtained one (for morphological and pharmacological experiments) or four (for electroresponsive properties) recordings. Since effect sizes could not be predicted before the experiments, we determined sample sizes by adopting common practices in the literature for similar experiments and erred on the side of oversampling to avoid Type II errors.

\section{RESULTS}

Prior studies suggest that, despite their low number, glutamatergic BNST-AV neurons might exert a disproportionate influence on behavior. Various factors might contribute to explain this paradoxical situation. Among them, the morphological properties of glutamatergic cells (dendritic extent or complexity) might allow them to integrate a higher number or more diverse set of inputs 
than GABAergic cells, they might have a higher intrinsic excitability, or they might be subjected to different neuromodulatory influences.

To explore these possibilities, we took advantage of the differential expression of the fluorescent reporter ZsGreen1 in the two mice crosses (Fig. 1) and obtained visually-guided wholecell recordings from 110 Vglut2-reporter-positive to 117 Vgatreporter-positive BNST-AV neurons.

Morphological properties of glutamatergic and GABAergic BNSTAV neurons

We recovered 39 biocytin-filled BNST-AV neurons (13 Vglut2reporter-positive; 26 Vgat-reporter-positive). Their morphology (Fig. 2) was first analyzed irrespective of transmitter phenotype. One cell type, termed "varicose neurons," was dominant, accounting for $56 \%$ of the cells. These neurons lacked dendritic spines but bore numerous dendritic varicosities distributed in a regular pattern, reminiscent of beads on a necklace (Fig. 2d, e, h). These varicosities had an ovoid shape $(\sim 2-4$ by $1-2 \mu \mathrm{m})$ and intervaricose segments were in the $2-7 \mu \mathrm{m}$ range. The incidence of these morphological properties was 77\% (10/13) among Vglut2reporter-positive cells and 46\% (12/26) among Vgat-reporterpositive cells (Fisher's exact test, $p=0.09$ ). It is unlikely that these peculiar morphological properties are artifacts of the slice preparation as they have also been observed before in tissue from perfused-fixed animals in the oval and juxtacapsular BNST regions, (see Figs. $7 \mathrm{C}$ and $16 \mathrm{~L}$ in ref. [26]).

A second type of cells, termed "spiny neurons," accounted for $34 \%$ of BNST-AV cells. These neurons exhibited a low density of dendritic spines that ranged in shape from thin to stubby (Fig. 2f, $g$, i-k). However, spines were not present in all dendritic segments. Moreover, although most of these cells bore dendritic varicosities, they lacked the regular necklace-like arrangement seen in the above cell type and were not present on all dendritic branches. Spiny neurons accounted for 35\% (9/26) of Vgat-reporter-positive cells but only $8 \%(1 / 13)$ of Vglut2-reporter-positive neurons (Fisher's exact test, $p=0.06$ ).

Thus, combining these morphological properties with transmitter phenotype yielded three main group of BNST-AV cells together accounting for $82 \%$ of recovered neurons: varicose and Vglut2reporter-positive (V-Glut; Fig. 2a), varicose and Vgat-reporterpositive (V-GAB; Fig. 2b), spiny Vgat-reporter-positive (S-GAB; Fig. 2c). Although the somata of S-GAB neurons were larger than that of V-GAB neurons (Table S1), somatic shapes overlapped extensively: round, oval, and angular somata were observed in the three cell groups. In contrast, there were differences in dendritic complexity and extent (Fig. $2 \mathrm{l}-\mathrm{n}$, see figure legend for statistics). Note that in the following quantification of these differences, we will use the term "process" rather than "dendrite" in acknowledgement of the fact that axon-like processes emerge from dendrites in $\sim 20 \%$ of BNST-AV neurons [25]. V-GAB cells had significantly fewer primary processes (Fig. 2l) and a lower process complexity overall (Fig. $2 \mathrm{~m}$ ) than V-Glut and S-GAB cells (see Table S1 for total dendritic length). Also, there was a trend for the process extent of V-Glut and V-GAB cells to be higher than that of $\mathrm{S}-\mathrm{GAB}$ cells (Fig. $2 \mathrm{n}$ ), but this analysis did not reach significance, probably due to dendrite truncation. Last, some V-Glut (Fig. 2A1) and V-GAB cells (Fig. 2b) had dorsally oriented dendritic branches that crossed the anterior commissure and extended in other BNST subnuclei. Of note, there were no differences in the distribution of physiological cell types among the V-GAB and S-GAB cells (Chisquare test, $X^{2}=1.19, p=0.55$ ).

Last, we compared the glutamatergic and GABAergic cells irrespective of their morphological classification. We found that glutamatergic cells have significantly more primary processes $(t(37)=2.16, p=0.04)$, higher total dendritic length $(t(37)=2.37$, $p=0.02)$ and higher overall process complexity $(t(37)=2.35$ $p=0.02$ ).
Glutamatergic and gabaergic ventral BNST neurons differ in their physiological properties...

NZ Gungor et al.

Electroresponsive properties of glutamatergic and GABAergic

2129

BNST-AV neurons

Previously, three main classes of BNST neurons were distinguished in the basis of their electroresponsive properties [25, 27] and mRNA expression for different ion channel subunits [28]. When depolarized, the two prevalent classes display a regular spiking (Type-I) or low-threshold bursting (Type-II) phenotype and both exhibit time-dependent inward rectification in the hyperpolarizing direction. A less common cell class (Type-III) lacks the last feature and instead exhibits fast inward rectification in response to hyperpolarization and a regular spiking phenotype when depolarized. Interestingly, the incidence of the three cell classes in BNSTAV neurons conformed with previous observations but did not differ between Vglut2-reporter-positive and Vgat-reporter-positive cells (Fig. 3a; Chi-square test, $X^{2}=1.2, p=0.5$ ).

While we did not find significant differences between the time constant and $R_{\text {in }}$ of Vglut2-reporter-positive and Vgat-reporterpositive neurons (Table S2), Vglut2-reporter-positive cells showed numerous signs of increased excitability (Fig. 3b-d, see figure legend for statistics). In response to juxta-threshold depolarizing current pulses, Vglut2-reporter-positive cells fired at shorter latencies (Fig. 3B1) and at a higher rate (Fig. 3B2) than Vgatreporter-positive neurons. In addition, when we examined responses to juxta-threshold current pulses, the membrane potential trajectory deviated from passive responses earlier and at more negative potentials in Vglut2-reporter-positive neurons (Figs. 3B4 and 5). At last, the depolarizing sag in voltage responses to hyperpolarizing current pulses had a significantly higher amplitude in Vglut2-reporter-positive cells (Fig. 3C1) and they exhibited higher firing rates during depolarizing current pulses, at all intensities tested (Fig. 3C2).

Consistent with these signs of increased excitability, spontaneous firing at rest was significantly more common in Vglut2reporter-positive (79\%) than Vgat-reporter-positive cells (53\%; ChiSquare test, $X^{2}=7.79, p=0.003$; Fig. $3 \mathrm{~d}$ ) and the spontaneously active cells had significantly higher firing rates in Vglut2-reporterpositive $(9.48 \pm 1.11 \mathrm{~Hz} ; n=42 / 53)$ than Vgat-reporter-positive $(4.19 \pm 0.84 \mathrm{~Hz} ; \quad n=27 / 51) \quad$ cells $\quad(t(67)=2.56, \quad p=0.01$, independent-samples $t$-test).

Noradrenergic modulation of glutamatergic transmission A number of studies have shown that NA is released in BNST-AV during stress (see Introduction) and that it exerts inhibitory effects on BNST-AV neurons [21, 22, 29-31]. However, in these earlier studies, the transmitter phenotype of recorded cells was not identified and, presumably, most of the sampled cells belonged to the prevalent group of GABAergic neurons. To investigate whether NA differentially modulates excitatory inputs to glutamatergic and GABAergic cells, we assessed NA modulation of ST-evoked EPSPs $(0.05 \mathrm{~Hz})$ in BNST-AV neurons manually clamped at $-55 \mathrm{mV}$, in the presence of picrotoxin $(100 \mu \mathrm{M})$. Importantly, no time-dependent changes in EPSP amplitudes were observed during the $10 \mathrm{~min}$ baseline period, combined across all pharmacological experiments described below (one-way repeated-measures ANOVA with 30 baseline data points per cell separated into 2 min bins; $F_{\text {glut }}(4,66)$ $\left.=1.94, p=0.1 ; F_{\text {gat }}(4,60)=2.15, p=0.08\right)$. Importantly, a mixedeffects ANOVA showed no interaction $\mathrm{F}_{\text {int }}(4,504)=0.831, p=0.51$, indicating that glutamatergic and GABAergic cells were not differentially affected by a potential rundown. To further ascertain that the effects caused by agonists are not contamined by a rundown, we repeated all the analysis explained below after excluding cells with baseline rundowns more than 25\% (Fig. S2). All the effects remained the same.

NA $(100 \mu \mathrm{M})$ caused a significant reduction of EPSP amplitudes in both Vglut2-reporter-positive ( $n=17$; control, $5.4 \pm 0.8 \mathrm{mV}$; NA, $3.3 \pm 0.4 \mathrm{mV} ; F_{\text {glut }}(2,32)=17.9 ; p=0.001$, one-way repeated-measures ANOVA with raw $5 \mathrm{~min}$ control, $5 \mathrm{~min}$ agonist, and $10 \mathrm{~min}$ values; Fig. 4a, blue) and Vgat-reporter-positive cells ( $n=14$; 
2130 A

\section{A}

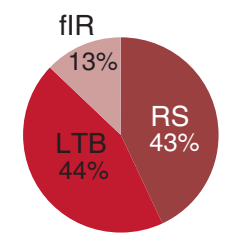

Physiological cell types

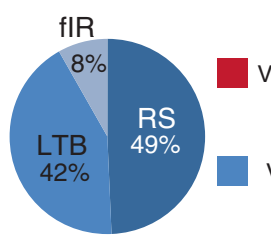

Vgat-reporter-positive

Vglut2-reporter-positive

Spiking at rheobase
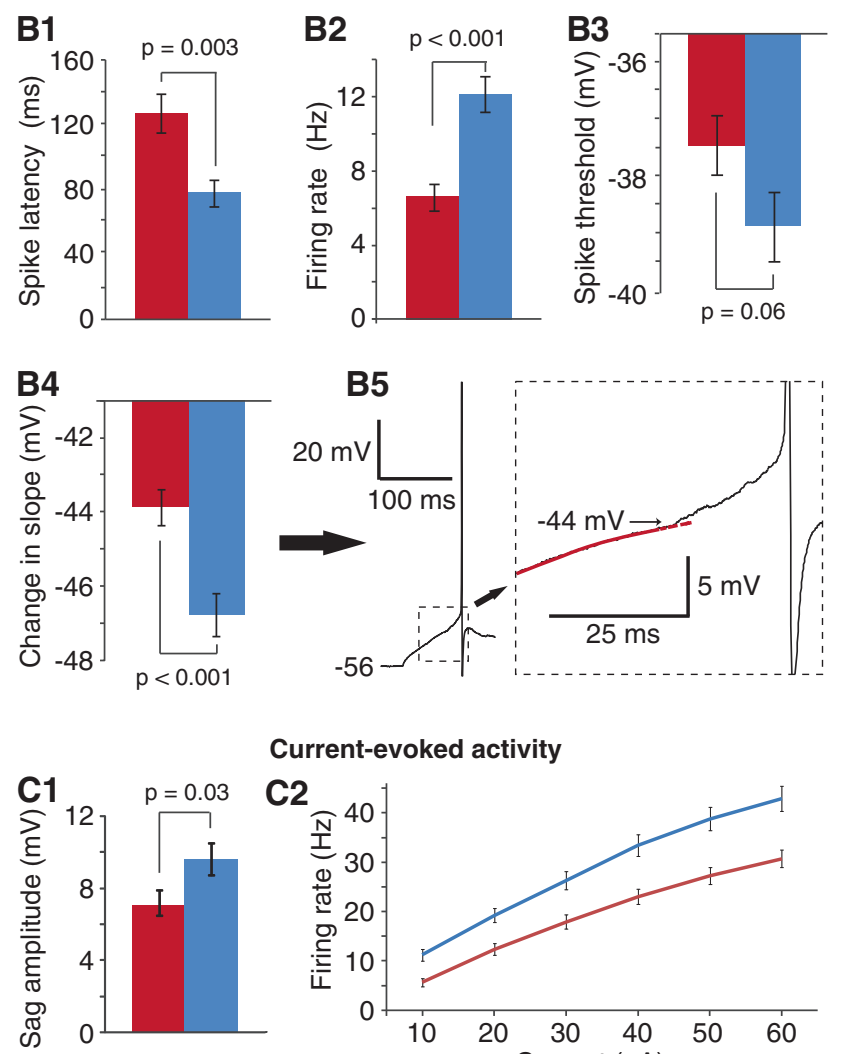

Current-evoked activity

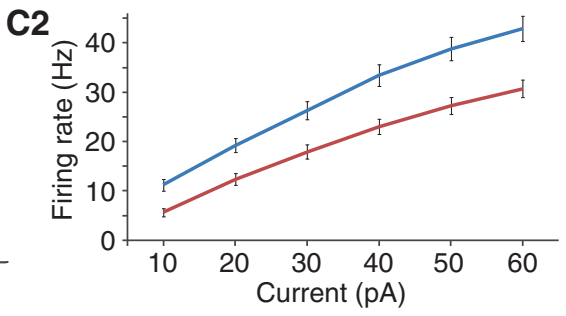

Spontaneous activity

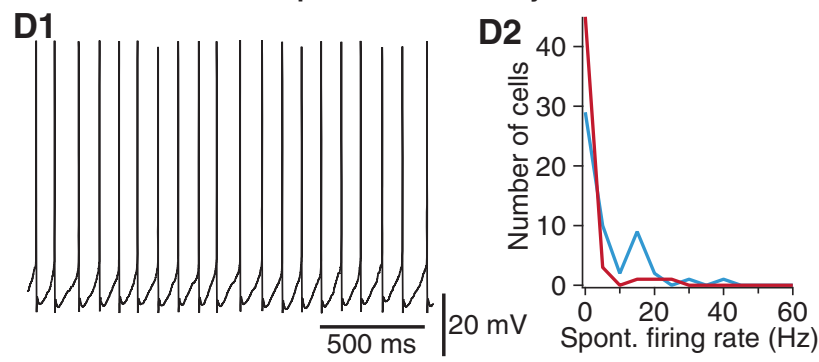

control, $6.2 \pm 0.6 \mathrm{mV}$; NA, $2.1 \pm 0.3 \mathrm{mV} ; F_{\text {gat }}(2,26)=52 ; p<0.001$; Fig. $4 \mathrm{a}$, red; see Fig. S1A for 2 min bins; see Fig. S2A for the same analysis only with cells with no baseline rundown). However, the NA-induced EPSP reduction was significantly less pronounced in Vglut2-reporter-positive cells (Vglut2-reporter-positive, $32 \pm 8 \%$ decrease; Vgat-reporter-positive, $64 \pm 6 \%$ decrease; mixed-effects ANOVA $F(2,58)=5.76, p=0.005$; see figure legend for statistics that compare all time points) and it was shorter-lasting. Indeed, the washout period did not fully restore EPSP amplitudes in the Vgat-reporter-positive cells whereas Vglut2-reporter-positive cells recovered within $10 \mathrm{~min}$ of return to the control superfusate.
Fig. 3 Physiological properties of Vglut2-reporter-positive and Vgatreporter-positive BNST-AV cells. A Incidence of physiological cell types among Vgat-reporter-positive (A, left) and Vglut2-reporterpositive (A, right) BNST-AV cells. B Differences between Vgatreporter-positive (red) and Vglut2-reporter-positive (blue) cells, assessed by independent-samples $t$-tests in $\mathbf{B 1}$ latency to fire at rheobase, $t(91)=3.1, p=0.003$, B2 firing rate at rheobase, $t(91)=$ 4.4, $p<0.001$, B3 spike threshold, $t(91)=1.9, p=0.06$, and B4 first change in slope before spike threshold $t(91)=4.2, p<0.001$. B5 Method used to detect the first change in slope before spike threshold. C1 Depolarizing sag amplitude with $-60 \mathrm{pA}$ current injection, $t(91)=2.1, p=0.03$. C2 Rate of firing ( $y$-axis) during depolarizing current pulses of increasing amplitude ( $x$-axis), $F_{\text {betw }}(1,80)=15.3, \quad p<0.001, \quad F_{\text {within }}(5,400)=470.3, \quad p<0.001$, $F_{\text {int }}(5,400)=7.1, p<0.001$, mixed-effects ANOVA. D1 A Vglut2reporter-positive cell at rest firing spontaneously at $8.5 \mathrm{~Hz}$. D2 Frequency distribution of the spontaneous firing rates of Vglut2reporter-positive (blue line) and Vgat-reporter-positive (red line) cells

We detected no evidence that the NA-induced inhibition of STevoked EPSPs resulted from post-synaptic changes in excitability. That is, we observed no consistent shift in the current injected to keep the cells at $-55 \mathrm{mV}$ and, in a separate subsets of cells, no change in firing rates at rheobase (Vglut2-reporter-positive: control, $7.9 \pm 1.7 \mathrm{~Hz} ; \mathrm{NA}, 9.3 \pm 2.9 \mathrm{~Hz}, t(12)=0.77, p=0.46$, paired $t$-test; Vgat-reporter-positive: control, $5.4 \pm 2.3 \mathrm{~Hz}$; NA, $6.5 \pm 2.1 \mathrm{~Hz}$, $t(6)=1, p=0.35)$. Moreover, NA did not alter the Rin of the Vglut2-reporter-positive cells ( $n=17$; control, $971 \pm 116 \mathrm{M} \Omega, \mathrm{NA}$, $\left.960 \pm 126 \mathrm{M} \Omega, F_{\text {glut }}(2,16)=1.3, p=0.3\right)$ while in the Vgat-reporterpositive cells, it produced a minor and short-lived reduction $(n=$ 14; control, $1234 \pm 169 \mathrm{M} \Omega, \mathrm{NA}, 1073 \pm 142 \mathrm{M} \Omega, 5$ min wash, 1204 $\left.\pm 181 \mathrm{M} \Omega ; F_{\text {gat }}(2,13)=4.8, p=0.02\right)$ that did not match the time course of the EPSP inhibition.

To identify which NA receptors mediate the differential EPSP modulation in Vglut2-reporter-positive and Vgat-reporter-positive cells, we tested the effects of a1 (Fig. 4c), a2 (Fig. 4d), and nonselective $\beta A R$ agonists (Fig. 4e) on ST-evoked EPSPs. For the three types of experiments, we provide the average $( \pm S E M)$ of all tested Vglut2-reporter-positive (blue) and Vgat-reporter-positive (red) cells (Fig. 4c-e) as well as the data obtained in individual cells (Fig. 4b).

The a1 receptor (a1AR) agonist phenylephrine $(10 \mu \mathrm{M})$ reduced EPSP amplitudes in both, Vglut2-reporter-positive ( $n=12$; control, $4.6 \pm 0.4 \mathrm{mV}$; phenylephrine, $3.6 \pm 0.4 \mathrm{mV} ; \quad F_{\text {glut }}(2,22)=9.4, p<$ $0.001)$ and Vgat-reporter-positive cells $(n=15$; control, $4.6 \pm 0.4$ $\mathrm{mV}$; phenylephrine, $3.5 \pm 0.7 \mathrm{mV} ; F_{\text {gat }}(2,28)=3.4, p=0.05$, Fig. $4 \mathrm{c}$, S1B, S2B). Similarly, the a2 receptor (a2AR) agonist UK 14-304 (1 $\mu \mathrm{M})$ reduced EPSP amplitudes in both, Vglut2-reporter-positive ( $n$ $=11$; control, $4.1 \pm 0.6 \mathrm{mV}$; UK14-304, $2 \pm 0.2 \mathrm{mV} ; F_{\text {glut }}(2,20)=14.1$, $p<0.001)$ and Vgat-reporter-positive cells ( $n=9$; control, $5.9 \pm 0.7$ $\mathrm{mV}$; UK14-304, $3.7 \pm 0.8 \mathrm{mV}$; $F_{\text {gat }}(2,16)=13.7, p<0.001$, Fig. $4 d$, S1C, S2C). In contrast, application of the non-selective $\beta A R$ agonist isoproterenol $(2 \mu \mathrm{M})$ did not affect EPSP amplitudes in Vgatreporter-positive cells ( $n=13$; control, $4.9 \pm 0.5 \mathrm{mV}$; Isoproterenol, $\left.5.2 \pm 0.7 \mathrm{mV} ; \quad F_{\text {gat }}(2,22)=0.5, p=0.63\right)$ while a trend toward enhancement was observed in Vglut2-reporter-positive cells ( $n$ $=15$; control, $4.5 \pm 0.5 \mathrm{mV}$; isoproterenol, $5.3 \pm 0.6 \mathrm{mV} ; F_{\text {glut }}(2,28)$ $=2.1, p=0.14$, Fig. 4e, S1D, S2D).

To further investigate whether $\beta A R s$ play a role in the NA modulation of Vglut2-reporter-positive cells, we tested the effects of NA in the presence of the $\beta A R$ antagonist propranolol $(5 \mu \mathrm{M}$, Fig. 5, S1E, S2A). When $\beta A R s$ were blocked, NA further reduced EPSP amplitudes in Vglut2-reporter-positive cells ( $\beta A R s$ not blocked: $n=17,32 \pm 8 \%$ decrease, $\beta$ ARs blocked: $n=12,62 \pm$ $6 \%$ decrease, $t(27)=2.9, p=0.007$, independent-samples $t$-test) but not in Vgat-reporter-positive cells ( $\beta$ ARs not blocked: $n=14$, $64 \pm 6 \%$ decrease, $\beta$ ARs blocked: $n=11,58 \pm 7 \%$ decrease, $t(23)=$ $0.7, p=0.49$ ). 

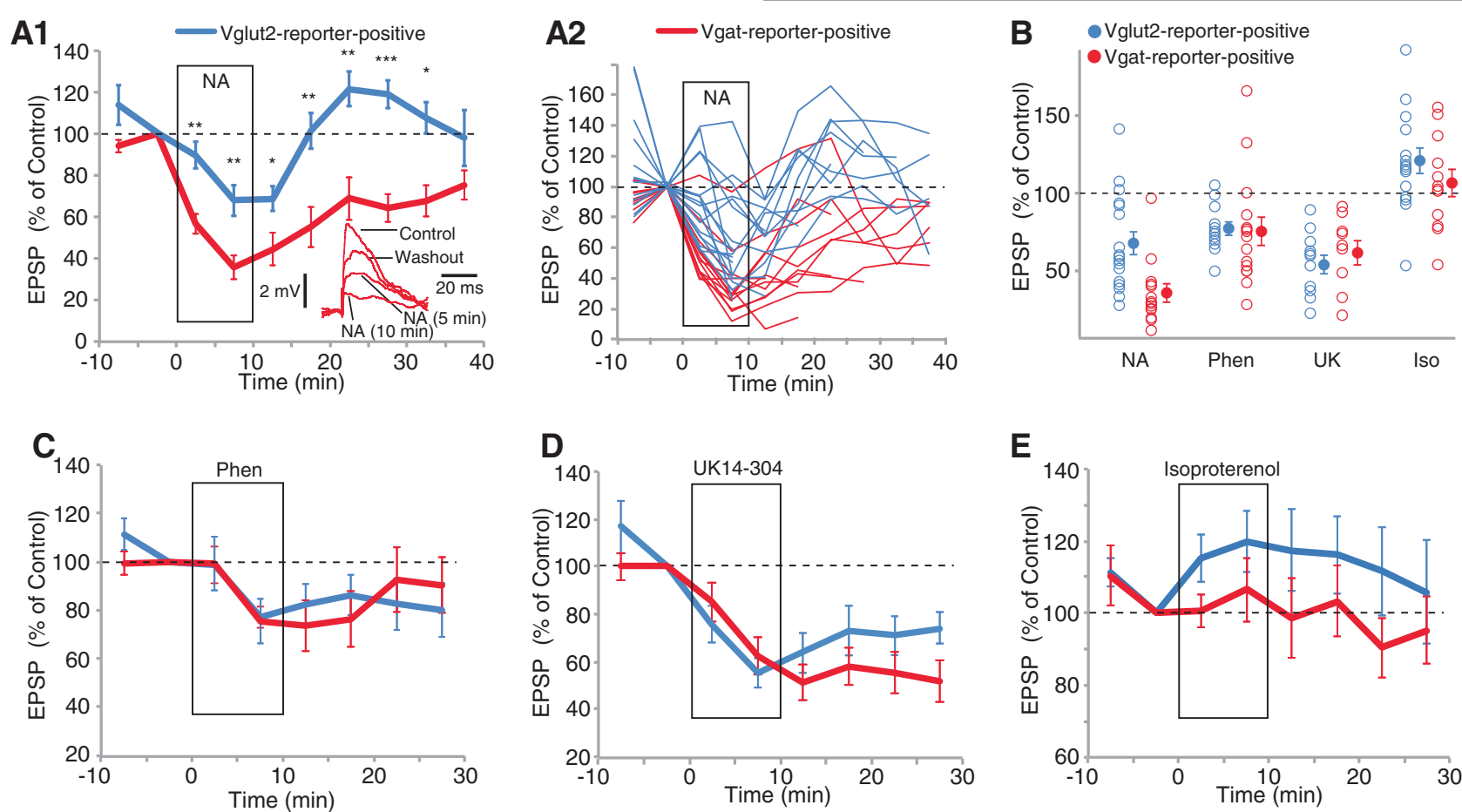

Fig. 4 Effects of NA receptor agonists on ST-evoked EPSPs in Vglut2-reporter-positive and Vgat-reporter-positive BNST-AV cells. A1, C, D, E show the average \pm SEM of all tested Vgat-reporter-positive (red) and Vglut2-reporter-positive (blue) cells. A Effects of NA (100 $\mu$ M). NA reduced EPSP amplitudes in both cell types. Inset, Example traces from a Vgat-reporter-positive cell. A1 Averages. A2 Individual cells. B Percent change in EPSP amplitudes after $5 \mathrm{~min}$ of agonist application. Data obtained in all experiments. C Effects of $\alpha 1$ receptor agonist phenylephrine $(10 \mu \mathrm{M})$. D Effects of $\alpha 2$ receptor agonist UK 14-304 $(1 \mu \mathrm{M})$. E Effects of $\beta$ AR agonist isoproterenol $(2 \mu \mathrm{M})$. Significant differences in EPSP amplitude change were observed between Vglut2-reporter-positive and Vgat-reporter-positive cells at all time points, tested with independent samples $t$-tests, as marked by stars $\left({ }^{*} p<0.05,{ }^{* *} p \leq 0.01,{ }^{* *} p \leq 0.001\right.$, Bonferroni-Holm corrected for multiple comparisons across time)

\section{DISCUSSION}

We compared the morpho-physiological properties and NA responsiveness of Vglut2-reporter-positive and Vgat-reporterpositive BNST-AV neurons. The interest of these comparisons stems from evidence that even though glutamatergic cells constitute a minute portion of BNST-AV neurons, they seem to exert an outsized influence over behavior. We found multiple properties that may contribute to the disproportionate influence of glutamatergic cells, including a slightly more complex morphology, higher intrinsic excitability, and different responsiveness to NA. Below, we consider the significance of these findings in the context of current knowledge about the anatomy and function of BNST-AV.

Anatomical properties of glutamatergic and GABAergic BNST-AV neurons

Besides a massive NA innervation from the brainstem A1 and A2 cell groups [14, 15], the main afferents to BNST-AV arise from the central and basolateral amygdala, insular cortex, subiculum, midline thalamic nuclei as well as various hypothalamic and brainstem autonomic nuclei. In turn, BNST-AV projects to the central amygdala, hypothalamus, VTA, and several brainstem autonomic nuclei. Through these connections, BNST-AV is thought to mediate anxiety and stress (reviewed in ref. [2]).

While there is much data about the connectivity of BNST-AV as a whole, it is unclear whether glutamatergic and GABAergic BNST-AV neurons form different connections. On the output side, early reports suggested that only glutamatergic BNST-AV cells project to the VTA [32], but later studies showed that GABAergic cells actually contribute most of this projection [4]. Similarly, glutamatergic and GABAergic neurons both project to other BNST sectors [33] and $\mathrm{CeA}$, although most of the latter projections are inhibitory [24]. Thus, both cell types contribute convergent projections to some of the areas targeted by BNST-AV. However, this may not be the case for all of BNST-AV's targets. Moreover, even in cases of convergent projections, the two cell types may contact different cell types.

On the input side, no tract-tracing studies have examined whether the two cell types receive different afferents. However, the distinct responsiveness of the two cell types to aversive and appetitive stimuli [7] is compatible with this possibility.

Physiological properties of glutamatergic and GABAergic BNST-AV neurons

Another factor that likely contributes to the outsized influence of glutamatergic cells is their higher intrinsic excitability. Supporting this possibility, we found that Vglut2-reporter-positive cells had a more negative firing threshold than Vgat-reporter-positive cells and that most $(\sim 80 \%)$ fired spontaneously at rest ( $\sim 10$ vs. $\sim 4 \mathrm{~Hz}$ for Vgat-reporter-positive cells). Moreover, when depolarized, Vglut2reporter-positive cells fired at shorter latencies and higher rates than Vgat-reporter-positive cells, even though their Rin was similar.

These disparities likely depend on the differential expression of one or more intrinsic voltage-gated currents. Indeed, neurons express diverse types of voltage-dependent ion channels whose differential distribution induces variability in the dynamics of current-evoked spiking and allows some types of neurons to fire spontaneously in an oscillatory manner in the absence of synaptic inputs [34]. For instance, spontaneous neuronal activity is commonly regulated by the hyperpolarization-activated mixed cation current $I_{\mathrm{H}}$ [35-37], the non-inactivating sodium persistent current INAP [38-40], and various types of potassium currents [41], all of which have been observed in BNST neurons $[25,27]$. Furthermore, RT-PCR studies have established that expression of the underlying channel sub-units varies between different physiological classes of BNST neurons [28]. 

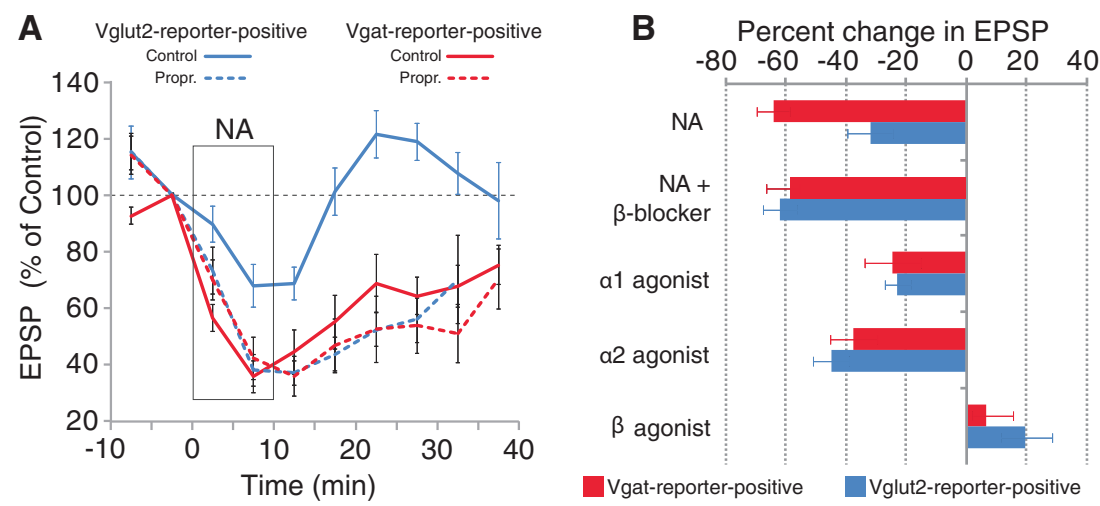

Fig. 5 Effects of NA in the presence of $\beta A R$ blocker propranolol. A Effect of NA in control conditions (solid lines) or in the presence of the $\beta A R$ blocker propranolol (dashed lines; $5 \mu \mathrm{M}$ ) in Vglut2-reporter-positive (blue) and Vgat-reporter-positive (red) BNST-AV neurons. B Summary of all pharmacological experiments

Thus, differences in the expression of voltage-gated channels might explain the differing excitability of glutamatergic and GABAergic neurons. For instance, we observed that during hyperpolarization, Vglut2-reporter-positive cells exhibit more pronounced depolarizing sags than Vgat-reporter-positive cells. Since $I_{\mathrm{H}}$ shifts the $V_{\mathrm{m}}$ positively during hyperpolarization, higher expression of $I_{\mathrm{H}}$ by glutamatergic cells might contribute to their faster recovery from spike after-hyperpolarizations. Similarly, higher expression of INAP or TRP-C [42] channels could be partly responsible for the higher excitability of glutamatergic cells. It will be important to compare the expression of these various ionic channels in glutamatergic and GABAergic BNST-AV neurons.

Differential noradrenergic regulation of glutamatergic and GABAergic BNST-AV neurons

Since BNST-AV receives one of the densest NA innervation in the brain and NA is released in BNST-AV during aversive events (see introduction), we compared the NA modulation of ST-evoked EPSPs in Vglut2-reporter-positive and Vgat-reporter-positive neurons. We found that the inhibition of EPSP amplitudes by NA was markedly less pronounced and shorter lasting in Vglut2-reporterpositive than Vgat-reporter-positive neurons $(\sim 30$ and $60 \%$ reduction, respectively). Previously, it was reported that NA inhibits glutamatergic transmission in BNST via the activation of a1ARs and a2ARs [22, 29-31, 43, 44] and the suppression of glutamate release [45]. Consistent with these findings, we found that NA reduces EPSP amplitudes in Vglut2-reporter-positive and Vgat-reporter-positive neurons and that a1AR and a2AR agonists mimic the inhibitory effects of NA.

Although prior electrophysiological studies reported that a1ARs and a2ARs mediate similar inhibitory effects in BNST neurons, their influence on behavior was found to differ. Intra-BNST infusions of a1 AR antagonists block anxiety-like behaviors induced by immobilization stress [17, 46] or aversive contexts [47], and reduce plasma levels of adrenocorticotropic hormone [17]. In contrast, intra-BNST infusions of a2AR agonists reduce freezing evoked by predator odor [18] and they lower light-enhanced and fear-potentiated startle [48]. Although a2AR underlie the most conspicuous physiological effect of NA, a2 receptors are also autoreceptors whose activation reduces both basal [49] and stress-induced [18] NA release in BNST. Presumably, this is why behavioral studies found that intra-BNST-AV infusions of a2AR agonists produce outcomes that would be expected from reduced NA levels.

Besides aARs, $\beta A R s$ in BNST also regulate negative states $[17,19,50]$. Yet, in vitro studies reported that $\beta A R$ agonists do not affect glutamatergic transmission in BNST-AV [22]. Our results suggest that this negative finding might be due to the high number of GABAergic cells sampled. Indeed, we observed that while $\beta A R$ blockade did not alter NA effects in Vgat-reporter- positive cells, it caused a further reduction of EPSP amplitudes in Vglut2-reporter-positive cells. Consistent with this, $\beta A R$ agonist application induced a moderate increase of EPSP amplitudes in Vglut2-reporter-positive but not Vgat-reporter-positive cells. Thus, our results suggest that the discrepancy between prior behavioral and physiological findings regarding the effects of $\beta A R s$ in BNST$A V$ results from the fact that $\beta A R s$ preferentially excite glutamatergic neurons.

\section{CONCLUSIONS}

Overall, our results suggest that several factors contribute to make glutamatergic cells more excitable than GABAergic BNST-AV neurons. Their dendrites ramify more extensively, potentially allowing them to integrate a higher number of inputs, they are intrinsically more excitable, driving many of them to fire spontaneously at high rates, and they are less inhibited by NA than their GABAergic neighbors. The differential NA modulation combined with differences in intrinsic excitability might position glutamatergic cells to dominate BNST-AV outputs in stressful conditions (but see ref. [13]).

Although the stress-inducing effects of $\beta A R s$ in BNST-AV might be explained by the excitation of the glutamatergic cells, the dominant effect of NA in BNST-AV is mediated by aARs and is inhibitory in both cell types. Since the glutamatergic cells stimulate the HPA-axis [8,9] and negative emotional states [7], NA's anxiogenic influence presumably depends on mobilizing glutamatergic cells. Possibly, the connectivity between glutamatergic and GABAergic cells supports network dynamics that increase the activity of glutamatergic cells and downstream effectors under NA modulation. Indeed, it is known that both glutamatergic and GABAergic cells make local connections, however these connections await characterization.

\section{ACKNOWLEDGEMENTS}

We would like to thank Bradford B. Lowell and Hongkui Zeng for making the transgenic lines used in this study available through Jackson labs. This material is based upon work supported by NIMH grant RO1 MH-098738 to Denis Paré.

\section{ADDITIONAL INFORMATION}

The online version of this article (https://doi.org/10.1038/s41386-018-0070-4) contains supplementary material, which is available to authorized users.

Competing interests: The authors declare no competing interests.

Publisher's note: Springer Nature remains neutral with regard to jurisdictional claims in published maps and institutional affiliations. 


\section{REFERENCES}

1. Walker DL, Miles LA, Davis M. Selective participation of the bed nucleus of the stria terminalis and CRF in sustained anxiety-like versus phasic fear-like responses. Prog Neuropsychopharmacol Biol Psychiatry. 2009;33:1291-308.

2. Gungor NZ, Pare D. Functional heterogeneity in the bed nucleus of the stria terminalis. J Neurosci. 2016;36:8038-49.

3. Daniel SE, Rainnie DG. Stress modulation of opposing circuits in the bed nucleus of the stria terminalis. Neuropsychopharmacology. 2016;41:103-25.

4. Kudo $T$, Uchigashima M, Miyazaki T, Konno K, Yamasaki M, Yanagawa $Y$, Minami $M$, Watanabe M. Three types of neurochemical projection from the bed nucleus of the stria terminalis to the ventral tegmental area in adult mice. J Neurosci. 2012;32:18035-46.

5. Poulin J, Arbour D, Laforest S, Drolet G. Neuroanatomical characterization of endogenous opioids in the bed nucleus of the stria terminalis. Prog Neuropsychopharmacol Biol Psychiatry. 2009;33:1356-65.

6. Nguyen AQ, Dela Cruz JA, Sun Y, Holmes TC, Xu X. Genetic cell targeting uncovers specific neuronal types and distinct subregions in the bed nucleus of the stria terminalis. J Comp Neurol. 2016;524:2379-99.

7. Jennings $J H$, Sparta DR, Stamatakis AM, Ung RL, Pleil KE, Kash TL, Stuber GD. Distinct extended amygdala circuits for divergent motivational states. Nature. 2013;496:224-8.

8. Radley JJ, Gosselink KL, Sawchenko PE. A discrete GABAergic relay mediates medial prefrontal cortical inhibition of the neuroendocrine stress response. J Neurosci. 2009;29:7330-40.

9. Radley JJ, Sawchenko PE. A common substrate for prefrontal and hippocampal inhibition of the neuroendocrine stress response. J Neurosci. 2011;31:9683-95.

10. Spencer SJ, Buller KM, Day T. Medial prefrontal cortex control of the paraventricular hypothalamic nucleus response to psychological stress: possible role of the bed nucleus of the stria terminalis. J Comp Neurol. 2005:481:363-76.

11. Choi DC, Furay AR, Evanson NK, Ostrander MM, Ulrich-Lai YM, Herman JP. Bed nucleus of the stria terminalis subregions differentially regulates hypothalamicpituitary-adrenal axis activity: implications for the integration of limbic inputs. J Neurosci. 2007;27:2025-34.

12. Crane JW, Buller KM, Day T. Evidence that the bed nucleus of the stria terminalis contributes to the modulation of hypophysiotropic corticotropinreleasing factor cell responses to systemic interleukin-1 $\beta$. J Comp Neurol. 2003;467:232-42.

13. Johnson SB, Emmons EB, Anderson RM, Glanz RM, Romig-Martin SA, Narayanan NS, LaLumiere RT, Radley JJ. A basal forebrain site coordinates the modulation of endocrine and behavioral stress responses via divergent neural pathways. J Neurosci. 2016;36:8687-99.

14. Fallon JH, Moore RY. Catecholamine innervation of the basal forebrain. J Comp Neurol. 1978;180:545-80.

15. Forray Ml, Gysling K, Andrés ME, Bustos G, Araneda S. Medullary noradrenergic neurons projecting to the bed nucleus of the stria terminalis express mRNA for the NMDA-NR1 receptor. Brain Res Bull. 2000;52:163-9.

16. Pacak K, McCarty R, Palkovits M, Kopin IJ, Goldstein DS. Effects of immobilization on in vivo release of norepinephrine in the bed nucleus of the stria terminalis in conscious rats. Brain Res. 1995;688:242-6.

17. Cecchi M, Khoshbouei, Javors M, Morilak DA. Modulatory effects of norepinephrine in the lateral bed nucleus of the stria terminalis on behavioral and neuroendocrine responses to acute stress. Neuroscience. 2002;112:13-21.

18. Fendt $M$, Siegl $S$, Streiniger-Brach B. Noradrenaline transmission within the ventral bed nucleus the stria terminalis is critical for fear behavior induced by trimethylthiazoline, a component of fox odor. J Neurosci. 2005;25:5998-6004.

19. Deyama S, Katayama T, Ohno A, Nakagawa T, Kaneko S, Yamaguchi T, Yoshioka M, Minami M. Activation of beta adrenoceptor protein kinase A signaling pathway within the ventral bed nucleus of the stria terminalis mediates the negative affective component of pain in rats. J Neurosci. 2008;28:7728-36.

20. Park J, Bucher ES, Fontillas K, Owesson-White C, Ariansen JL, Carelli RM, Wightman RM. Opposing catecholamine changes in the bed nucleus of the stria terminalis during intracranial self-stimulation and its extinction. Biol Psychiatry. 2013;74:69-76.

21. Casada H, Dafny N. Responses of neurons in bed nucleus of the stria terminalis to microiontophoretically applied morphine, norepinephrine and acetylcholine. Neuropharmacology. 1993;32:279-84.

22. Egli RE, Kash TL, Choo K, Savchenko V, Matthews RT, Blakely RD, Winder DG. Norepinephrine modulates glutamatergic transmission in the bed nucleus of the stria terminalis. Neuropsychopharmacology. 2005;30:657-68.

23. Vong L, Ye C, Yang Z, Choi B, Chua S, Lowell BB. Leptin action on GABAergic neurons prevents obesity and reduces inhibitory tone to POMC neurons. Neuron . $2011 ; 71: 142-54$
Glutamatergic and gabaergic ventral BNST neurons differ in their physiological properties...

NZ Gungor et al.

24. Gungor NZ, Yamamoto R, Pare D. Optogenetic study of the projections from the 2133 bed nucleus of the stria terminalis to the central amygdala. J Neurophysiol. 2015;114:2903-11.

25. Rodriguez-Sierra OE, Turesson HK, Pare D. Contrasting distribution of physiological cell types in different regions of the bed nucleus of the stria terminalis. J Neurophysiol. 2013;110:2017-49.

26. Larriva-Sahd J. Histological and cytological study of the bed nuclei of the stria terminalis in adult rat. II. Oval nucleus: extrinsic inputs, cell types, neuropil, and neuronal modules. J Comp Neurol. 2006;497:772-807.

27. Hammack SE, Mania I, Rainnie DG. Differential expression of intrinsic membrane currents in defined cell types of the anterolateral bed nucleus of the stria terminalis. J Neurophysiol. 2007;98:638-56.

28. Hazra R, Guo JD, Ryan SJ, Jasnow AM, Dabrowska J, Rainnie DG. A transcriptomic analysis of type I-III neurons in the bed nucleus of the stria terminalis. Mol Cell Neurosci. 2011;46:699-709.

29. Krawczyk M, Georges F, Sharma R, Mason X, Berthet A, Bezard E, et al. Double dissociation of the catecholoaminergic modulation of synaptic transmission in the oval bed nucleus of the stria terminalis. J Neurophysiol. 2011;105:145-53.

30. Matsui $\mathrm{H}$, Yamamoto $\mathrm{C}$. The possible involvement of adenylate cyclase inhibition in the field potential suppression through alpha-2 adrenergic receptors in the bed nucleus of the stria terminalis. Brain Res. 1984;293:197-190.

31. Sawada S, Yamamoto C. Postsynaptic inhibitory actions of catecholamines and opioid peptides in the bed nucleus of the stria terminalis. Exp Brain Res. 1981;41:264-70.

32. Georges F, Aston-Jones G. Activation of ventral tegmental area cells by the bed nucleus of the stria terminalis: a novel excitatory amino acid input to midbrain dopamine neurons. J Neurosci. 2002;22:5173-87.

33. Turesson HK, Rodriguez-Sierra O, Pare D. Intrinsic connections in the anterior part of the bed nucleus of the stria terminalis. J Neurophysiol. 2013;109:2438-50.

34. Llinás RR. The intrinsic electrophysiological properties of mammalian neurons: insights into central nervous system function. Science. 1988;242:1654-64.

35. Forti L, Cesana E, Mapelli J, D'Angelo E. Ionic mechanisms of autorhythmic firing in rat cerebellar Golgi cells. J Physiol. 2006;574:711-29.

36. He C, Chen F, Li B, Hu Z. Neurophysiology of HCN channels: from cellular functions to multiple regulations. Prog Neurobiol. 2014;112:1-23.

37. McCormick DA, Pape HC. Properties of a hyperpolarization-activated cation current and its role in rhythmic oscillation in thalamic relay neurones. J Physiol. 1990;431:291-318.

38. Bean BP. The action potential in mammalian central neurons. Nat Rev Neurosci. 2007;8:451-65.

39. Bevan MD, Wilson CJ. Mechanisms underlying spontaneous oscillation and rhythmic firing in rat subthalamic neurons. J Neurosci. 1999;19:7617-28.

40. Jackson AC, Yao GL, Bean BP. Mechanism of spontaneous firing in dorsomedial suprachiasmatic nucleus neurons. J Neurosci. 2004;24:7985-98.

41. Hille B. Ion channels of excitable membranes. Sunderland, MA: Sinauer; 2001.

42. Zhou FM, Lee CR. Intrinsic and integrative properties of substantia nigra pars reticulate neurons. Neuroscience. 2011;198:69-94.

43. McElligott ZA, Winder DG. a1-Adrenergic receptor-induced heterosynaptic longterm depression in the bed nucleus of the stria terminalis is disrupted in mouse models of affective disorders. Neuropsychopharmacology. 2008;33:2313-23.

44. Shields AD, Wang $Q$, Winder DG. alpha2A-adrenergic receptors heterosynaptically regulate glutamatergic transmission in the bed nucleus of the stria terminalis. Neuroscience. 2009;163:339-51.

45. Forray MI, Bustos G, Gysling K. Noradrenaline inhibits glutamate release in rat bed nucleus of the stria terminalis: in vivo microdialysis studies. J Neurosci Res. 1999;55:311-20.

46. Morilak DA, Cecchi M, Khoshbouei $H$. Interactions of norepinephrine and galanin in the central amygdala and lateral bed nucleus of the stria terminalis modulate the behavioral responses to acute stress. Life Sci. 2003;73:715-26.

47. Hott SC, Gomes FV, Fabri DRS, Reis DG, Crestani CC, Correa FMA, Resstel LBM Both alpha 1 and beta 1 adrenoceptores in the bed nucleus of the stria terminalis are involved in the expression of conditioned contextual fear. $\mathrm{Br} J$ Pharmacol. 2012;167:207-21.

48. Schweimer J, Fendt M, Schnitzler HU. Effects of clonidine injections into the bed nucleus of the stria terminalis on fear and anxiety behavior in rats. Eur J Pharmacol. 2005;507:117-24.

49. Forray MI, Bustos G, Gysling K. Regulation of norepinephrine release from the rat bed nucleus of the stria terminalis: in vivo microdialysis studies. J Neurosci Res. 1997;50:1040-6.

50. Vranjkovic O, Gasser PJ, Gerndt CH, Baker DA, Mantsch JR. Stress induced cocaine seeking requires a beta- 2 adrenergic receptor regulated pathway from ventral bed nucleus of the stria terminalis that regulates CRF actions in the ventral tegmental area. J Neurosci. 2014;34:12504-14. 